\title{
Competitive Binding in Mixed Surfactant Systems for Single-Walled Carbon Nanotube Separation
}

\author{
Rishabh M. Jain, ${ }^{\dagger}$ Micha Ben-Naim, ${ }^{\ddagger}$ Markita P. Landry, ${ }^{\ddagger}$ and Michael S. Strano ${ }^{*} \neq$ \\ ${ }^{\ddagger}$ Department of Chemical Engineering and ${ }^{\dagger}$ Materials Science Engineering, Massachusetts Institute of Technology, 77 Massachusetts \\ Avenue, Cambridge, Massachusetts 02139, United States
}

\section{Supporting Information}

ABSTRACT: The separation of single-walled carbon nanotubes (SWNTs) by chirality is of great interest to enable the next generation of optical and optoelectronic devices. Many separation schemes employ the surfactant sodium dodecyl sulfate (SDS), with or without a bile salt surfactant such as sodium cholate (SC). In this study, we observe and explain the effect of these mixed surfactant systems on the hydrogel-based selective adsorption separation method. We find that sodium cholate outcompetes SDS more effectively on smaller diameter tubes and quantify this difference as the sodium cholate concentration is increased and $(6,5)$ separation is diminished. These changes in separation efficiency with surfactant composition are understood using a theoretical model developed previously and predict that surfactant mixtures alter the charge per unit length of specific $(n, m)$ SWNTs, altering the separation. This understanding of the chiral dependence of the surfactant binding will not only enable a greater understanding of surfactant coverage on the SWNT but also pave the path to further control the SWNT separation processes that depend on these surfactants.
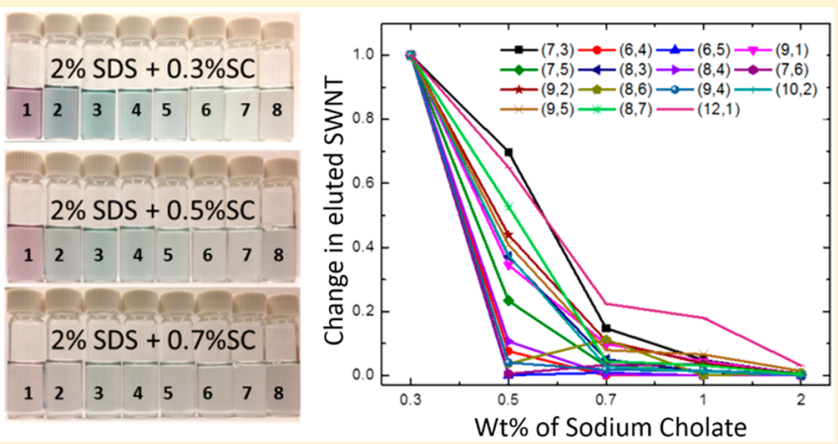

$\mathrm{T}$ he separation of single-walled carbon nanotubes (SWNTs) by chirality has gained immense interest in recent years across many fields with significant advances that enable both high purity and high yield separation of carbon nanotubes. ${ }^{1-16}$ These efforts from both our group and others have clearly demonstrated the ability to separate single chirality SWNTs at scales that are now enabling the next generation of nanotube-based optical sensors ${ }^{17-24}$ and optoelectronics. ${ }^{6,25-30}$ One of the first examples was shown by our group, where we demonstrated a bulk single chirality $(6,5)$ nanotube-based active layer for a near-infrared photovoltaic (6) and a ratiometric $(6,5)$ and $(7,6)$ SWNT-based optical sensor for in vivo imaging. ${ }^{20}$ The improved efficiency driven by the homogeneous electronic structure was corroborated by the Arnold group with a demonstration of a near single chirality $(7,5)$ photovoltaic. ${ }^{26}$ Among the several methods that exist to separate carbon nanotubes, advances in the gel-based separation, ${ }^{1,2,11-23,31,32}$ and more recently a two-phase method pioneered by the Zheng group, 9 seem to provide a promising path toward enabling industrial scale separations. Additional work by the Pang group has made significant advances in achieving a high dispersion of $(6,5)$ SWNTs using $\pi$-conjugated polymers and helically binding polymers for length-specific SWNT fractions. ${ }^{3,34}$ Mechanisms to understand these separations have also been proposed in order to help guide higher purity and yield separation processes. , $2,9,13,35-37^{\text {In all }}$ cases, it is well agreed upon that the role of surfactants in these processes is instrumental. In this study, we explore experimentally for the first time the impact of using different surfactants and surfactant mixtures in the gel-based separation. We show that the addition of bile salt surfactants modifies the chiralities of SWNTs that separate, showing a chiralitydependent binding of these surfactants on SWNTs.

In previous work we have developed a quantitative model that shows the sodium dodecyl sulfate (SDS) charge state is the most likely reason for chirality-based separation, and we expand upon this model herein for mixed-surfactant systems. ${ }^{1}$ Furthermore, our group ${ }^{1}$ and others ${ }^{13}$ have shown that in the range of $0.5-5 \mathrm{wt} \% \mathrm{SDS}$ in aqueous solution, nanotubes exhibit different surfactant coverage levels which affect their binding affinity to the gel. Other studies from the Kataura, Doorn, and other groups related to altering the SDS phase around the tube have used temperature, ${ }^{11} \mathrm{pH}^{35,38}$ and salt ${ }^{1,39}$ to manipulate the SDS phase around the tube, and most of these studies agree with our previous findings. ${ }^{1}$ These studies also indicate that the adsorption occurs directly between the SWNT surface and the Sephacryl, where the surfactant mediates the chiral selectivity but not the binding itself. ${ }^{1,31}$

An early high throughput study ${ }^{40}$ of various surfactants guided researchers to heavily rely on SDS. We expect this is due to the flexible morphology of the surfactant on the tube.

Received: August 14, 2015

Revised: September 3, 2015

Published: September 3, 2015 
$0.5 \%$ SDS

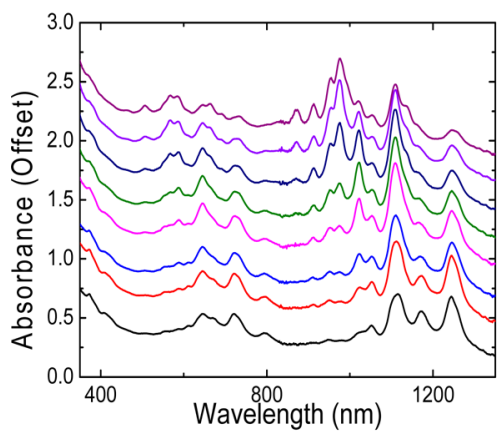

$0.5 \%$ SDOC

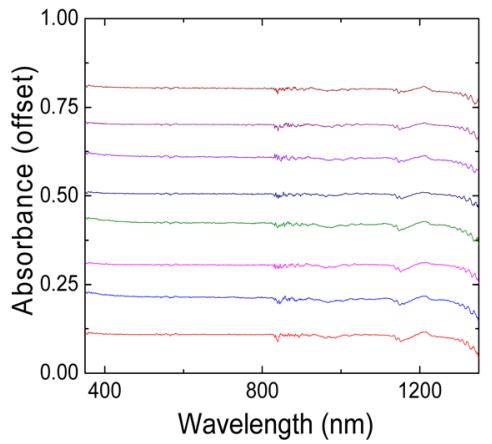

$0.5 \% \mathrm{SC}$

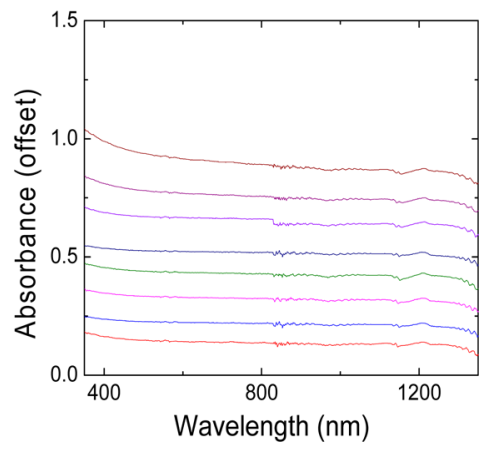

Figure 1. From left to right, the desorption spectra of several columns of SWNTs suspended in 0.5 wt \% of SDS, SDOC, and SC. The spectra indicate that SDS is the only surfactant that allows SWNTs to adsorb to the gel. Interestingly SC seems to allow some amorphous carbon to bind and desorb from the gel, as indicated via the sloping baseline. However, no SWNTs bind in just SC or SDOC surfactant suspensions.
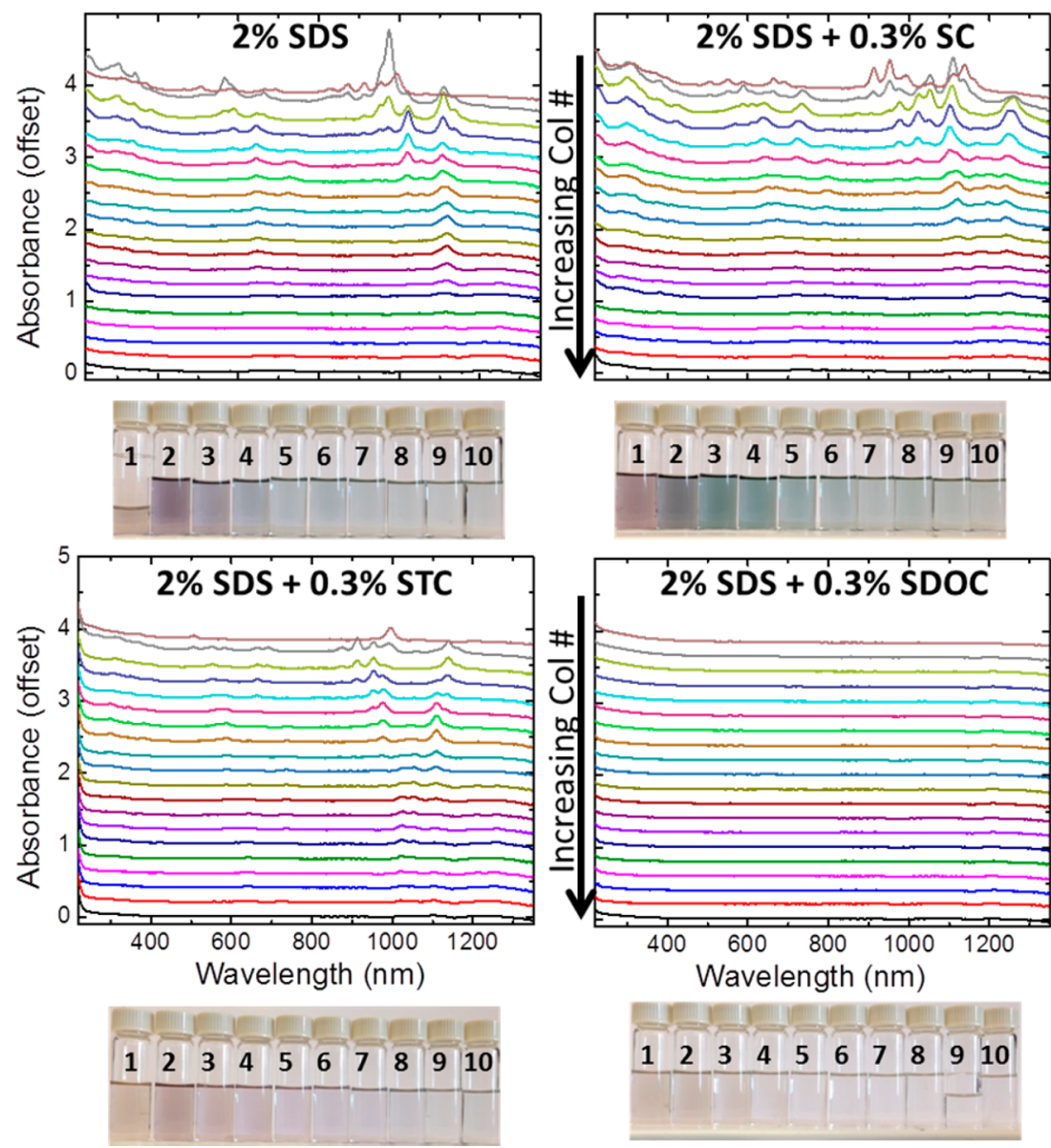

Figure 2. Absorbance spectra of 20 columns of eluted SWNTs that were adsorbed to the gel in surfactant solutions noted in the header of each plot area. Under each absorbance spectrum is a photograph of the first 10 columns of eluted SWNT, showing clearly visible differences in the quantities and purities of the SWNT solutions. Interestingly, the three bile salt surfactants show dramatically different separations despite small chemical moiety differences. Note that the addition of even a small amount of SDOC prevents any SWNT adsorption.

However, other methods, such as density gradient centrifugation $^{3-5,41,42}$ and the two-phase separation method, ${ }^{9,10}$ have found success via the use of surfactant mixtures. A recent study on the mechanism of the two-phase separation shows that the use of surfactant mixtures most heavily affects the coverage on the surface of the nanotube by each surfactant. ${ }^{43}$ The use of a bile salt surfactant creates a more tightly bound structure on the surface of the tube which leaves a lower fraction of the surface of the tube exposed. ${ }^{44}$ In the two-phase method this phenomenon is exhibited quite clearly; an increase in the sodium cholate concentration makes the nanotubes more hydrophilic as less of the tube surface is exposed. .,10,43 $^{-1}$

In this study we use these same surfactant coverage guiding principles to establish methods of using mixed surfactants in the separation of carbon nanotubes via the gel-based method. We first establish the protocol used to enable various monosurfactant separations. We then study the concentrationdependent effect of mixing various bile salt surfactants with 

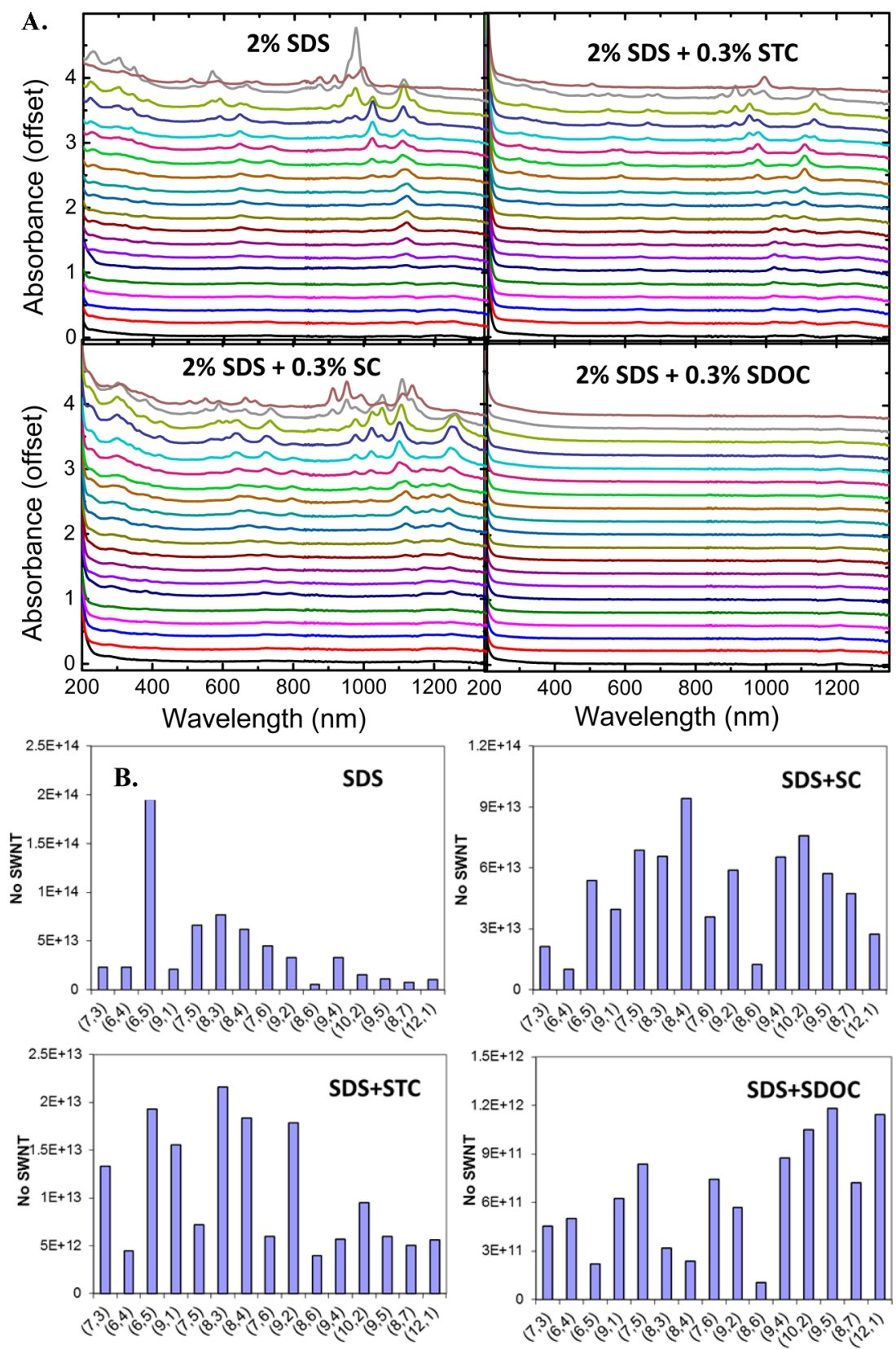

Figure 3. Absorbance spectra of 20 columns of separation with the addition of $0.3 \%$ bile salts. (A) Absorbance spectra of 20 columns of eluted SWNTs that were adsorbed to the gel in surfactant solutions noted in the header of each plot area. Interestingly, the three bile salt surfactants show dramatically different separations despite small chemical moiety differences. Note that the addition of even a small amount of SDOC prevents any SWNT adsorption. (B) Chiralities eluted over all 20 columns worth of the separation for each of the surfactant mixtures. The bars make it obvious which SWNT chiralities are preferentially absorbed to the gel. As is expected, the $(6,5)$ tube is most strongly absorbed and eluted in the SDS-only case. Note also that the SDOC absolute levels of SWNTs are within the noise limit of detection by our spectrometer, and we do not believe there are any measurable quantities of SWNTs eluted with the addition of SDOC.

SDS. We studied the four most commonly used surfactants for these systems, sodium dodecyl sulfate (SDS), sodium cholate (SC), sodium deoxycholate (SDOC), and sodium taurocholate (STC). We implicitly use our previously developed quantitative model $^{1}$ and the methods developed in those works to understand the experiments that we perform with both the single and mixed surfactant systems. This study will help outline a path to not only understand the gel-based separation processes further but also help understand how surfactant mixtures compete for SWNT coverage and their chiral dependence.

\section{SINGLE SURFACTANT SYSTEMS}

The first study that we perform is with single surfactant systems of three of the four surfactants of interest. We study various concentrations of each of the surfactants of interest, SDS, SC, and SDOC. We choose these surfactants because of their common usage in separation protocols. ${ }^{3,4,9,40,44}$ The bile salt surfactants (SC, SDOC, and STC) are all very similar to each other with a single-point mutation in the chemical structure. ${ }^{44}$ However, these minor differences cause major differences in the surfactant coverage on the SWNT. Unfortunately, STC is only commercially sold in small quantities, so creating a $100 \mathrm{~mL}$ 


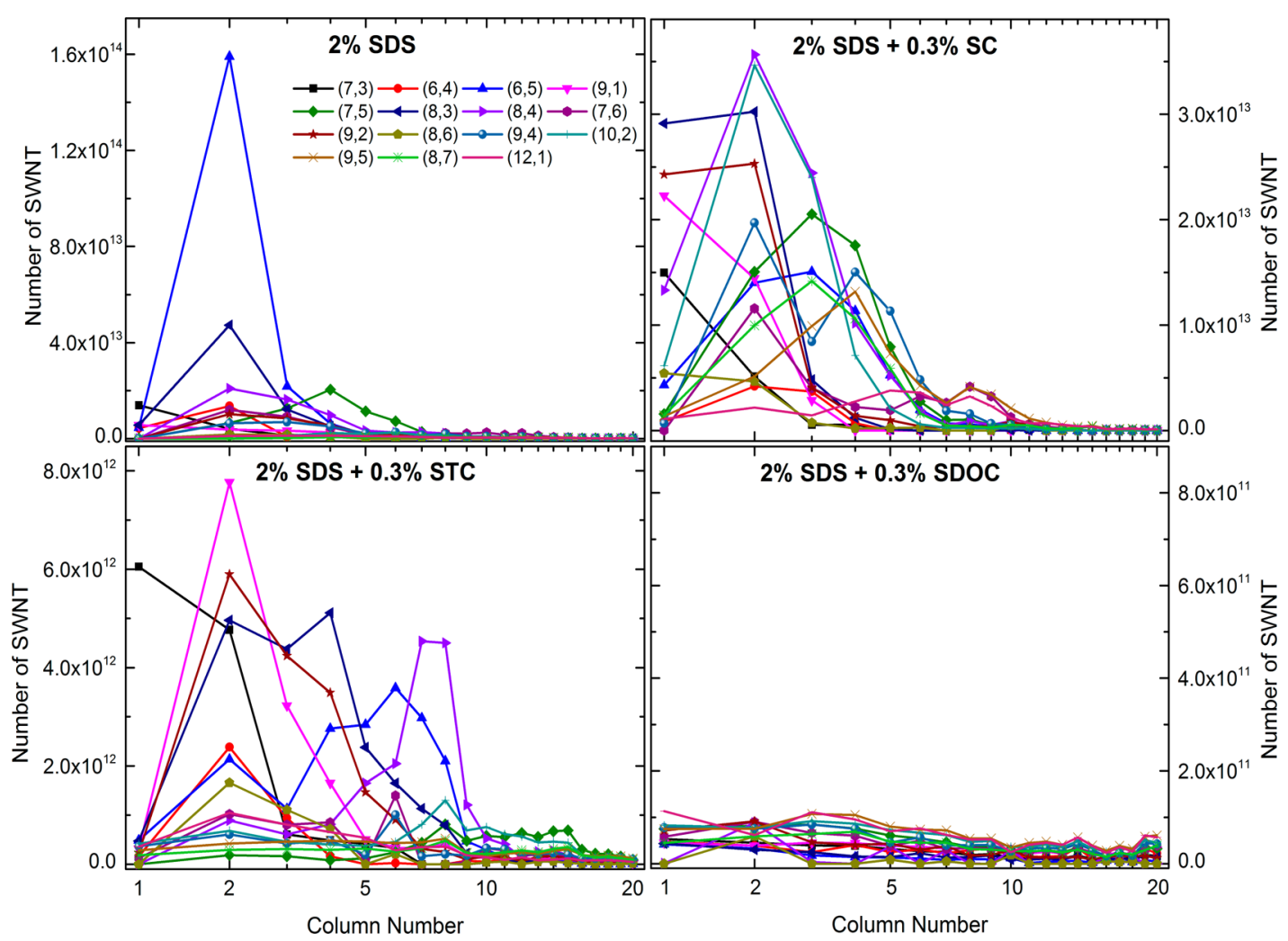

Figure 4. Calculated quantities of SWNT per column per chirality of 20 columns of eluted SWNTs for each surfactant mixture, plotted with a cumulative Gaussian $x$-axis for clarity. The amount of nanotubes changes dramatically for each surfactant mixture, and hence each graph has a different $y$-axis. We specifically note that the SC addition enables several large diameter chiralities to be eluted within the first 5 columns. Further, it is obvious that the $(6,5)$ tube is most efficiently eluted in the SDS-only elution. Finally, the addition of $0.3 \%$ STC shows in column 1 an elution of only the $(7,3)$ tube.

SWNT sample that is comparable to our other samples is difficult. Further, our mixed surfactant experiments show that it is highly unlikely that any SWNT would adsorb when using this surfactant alone.

We run the separation process with each of these surfactants and analyze the eluted content. In order to analyze the separation process, we run eight columns of the separation and take absorbance spectra of the eluted sample. Figure 1 shows spectra for an adsorption with each surfactant at $0.5 \%$ by weight solution of the respective surfactant.

The SWNT suspensions with a surfactant other than SDS do not show any SWNT adsorption or desorption. This result agrees with previous observations that SDS is unique in its ability to enable the separation of SWNTs based on chirality. ${ }^{1,13,40}$ We agree with the hypothesis that SDS has this ability due to its flexible morphology and equilibrium coverage ratios that are chirality and concentration dependent. The partial coverage of SDS of the SWNT enables part of the SWNT surface to stay exposed and therefore adsorb to the gel, ${ }^{1,38,39}$ or in the two-phase method, a dynamic and controllable hydrophobicity. $9,10,43$

Our previous work shows both experimentally and via a quantitative model that SDS has a chirality- and concentrationdependent morphology and coverage on the SWNT surface. ${ }^{1}$ We learned that at low SDS concentrations the surfactant has a low coverage on the tube and a lower effective charge per unit length. Further, we learned that larger diameter semiconducting tubes show greater SDS coverage than smaller diameter tubes at the same bulk SDS concentration. This understanding enables us to now interrogate the SWNT-surfactant system with other surfactants as well as mixed surfactants.

Despite a lack of adsorption when suspended in SC or SDOC alone, several groups have shown that mixing surfactants can be used to fine-tune the separation, especially when using the density gradient ${ }^{3,5,42}$ or two-phase methods. ${ }^{10,43}$ A systematic study of mixed surfactants in the gelbased system is presented here for the first time.

\section{MIXED SURFACTANT SYSTEMS}

We perform the surfactant mixture study via the creation of mixtures of SDS with one of the bile salt surfactants at experimentally relevant concentrations of $0-2 \%$ by weight of each. The first concentration mixture that we attempt is to add a small fraction, $0.3 \mathrm{wt} \%$, of the bile salt surfactant to an otherwise standard separation process at 2 wt \% SDS concentration. We carry out the separation process for 20 columns in order to ensure that we reach the point at which we no longer see any SWNT adsorbed to the gel. Figure 2 shows the spectra and images of the desorbed SWNT from the 20 columns on descending order, where the SWNT was eluted using 5 wt \% SDS.

As is shown in the spectra in Figure 2, the addition of a bile salt surfactant to SDS significantly alters the separation of the SWNT. The SDS-only separation looks similar to what has been shown in previous work, by both our group and others. The addition of either STC or SC does not prevent the separation but changes it. The addition of SDOC however does completely prevent the separation. This lack of separation implies that we can say with certainty that the bile salt 
surfactants outcompete the SDS binding on the SWNT to varying levels. It especially showcases that SDOC binds the SWNT the most tightly, as has been shown by our group and others.

We notice two important trends in the STC and SC additions. The SC addition enables and promotes the separation of larger diameter SWNTs, in a way similar to reducing the SDS concentration. On the basis of our previous work, we expect that if larger diameter SWNTs can be adsorbed to the gel then it implies that the SDS is not tightly packed on the SWNT. We expect that the SC additive is binding tightly on the SWNT; however, it does not completely outcompete the SDS. The ability to separate some SWNTs and especially larger diameter SWNTs implies that the SC allows the SDS to partially cover all chiralities of SWNTs, including the large diameters.

The total number of separable SWNTs in both cases (SDS alone and SDS $+0.3 \% \mathrm{SC}$ ) is similar. In fact, as was the case with a lower SDS concentration, the mixed surfactant enables more SWNTs to get bound $\left(7.3 \times 10^{14}\right.$ SWNT $)$ over the 20 columns than just SDS at $2 \%\left(6.3 \times 10^{14} \mathrm{SWNT}\right)$. The absorption spectra for these separations are shown in Figure $3 \mathrm{~A}$, while Figure $3 \mathrm{~B}$ shows the preferential adsorption of various SWNT chiralities over the 20 columns of separation performed. Figure 4 shows the quantities of each SWNT eluted for every column when STC, SDOC, and SC are added to the SDS SWNT mixture. Further, Figure 1 showcases that the chiralities of SWNTs are also similar between a $0.5 \%$ SDS and the $0.3 \%$ SC addition to the $2 \%$ SDS solution. This implies that the addition of a small quantity of SC reduces the overall surface coverage of the SDS surfactant on the SWNT. While a molecular picture cannot be precisely drawn from this observation, we expect that the SC additive influences the morphology of the SDS to assume a loose packing that enables more SWNTs and larger diameter SWNTs to adsorb to the gel.

The STC addition in contrast to the SC addition has a chiral selectivity that enables the separation of SWNTs specifically with absorbance peaks close to 1250 and $950 \mathrm{~nm}$. We further analyzed the spectra to determine specifically which SWNTs are adsorbed and eluted with this surfactant mixture as seen in Figure 4. We find that the $(7,3),(6,5),(9,1),(8,3),(8,4)$, and $(9,2)$ SWNTs are preferentially adsorbed over the 20 columns of separation performed as shown in Figure 4. All of these chiralities have diameters between 0.7 and $0.8 \mathrm{~nm}$. In fact, column 1 has over $80 \%$ pure $(7,3)$.

Further, the total number of SWNTs that are adsorbed to the gel over the 20 columns is much smaller $\left(1.6 \times 10^{14}\right.$ SWNT) than the SDS or SDS plus SC experiments. The selectivity is very different than what has been seen previously by our group and others. ${ }^{1,12,13,35}$ These observations imply that the STC binds certain chiralities tighter than others at the same bulk STC concentration. We expect what we observe in our experiments is that the SDS can outcompete the less tightly bound STC chiralities, which enables their adsorption and desorption to and from the gel. This observation seems to suggest that these small diameter SWNTs have a lower binding affinity to the STC than other chiralities. This chiralitydependent binding of STC was previously shown in work from our group in the context of reactions with aryl diazonium salts. ${ }^{44}$

Given the significant changes in separation, we investigated whether our previously developed quantitative model could be applied to this system. ${ }^{1,2}$ We provide a model for a two- surfactant system based on a modified model for SDS-mediated adsorption and desorption of SWNTs to a sephacryl binding site. The initial binding of a semiconducting SWNT to a binding site can be energetically modeled by outlining the total system energy $V(d)$ as a function of the distance between the sephacryl site and the SWNT $(d)$. The van der Waals forces balance to describe this system energy as follows

$$
V(d)=V_{\text {van der Waals }}(d)-V_{\text {electrostatic }}(d)-V_{\text {hard sphere }}(d)
$$

where $V_{\text {electrostatic }}$ is the electrostatic repulsive force; $V_{\text {hard surface }}$ is the hard-surface repulsive force; and $V_{\text {van der Waals }}$ is the attractive force. Subsequently, we expand the description of the van der Waals energy to describe the attractive force between an available sephacryl binding site and a semiconducting SWNT, where the former is approximated as an anisotropic planar surface and the latter as a SWNT with a variable radius as defined by its chirality. ${ }^{45}$

$$
\begin{gathered}
V_{\text {van der Waals }}(d)=\left[-\frac{\sqrt{2 r_{\text {SWNT }}}}{24 d^{3 / 2}}\left(\frac{3}{2} H_{\mathrm{N}}\right)\left(\frac{1}{1+e^{\beta\left(d-d_{0}\right)}}\right)\right. \\
\left.-\frac{r_{\text {SWNT }}^{2}}{6\left(d+r_{\text {SWNT }}\right)^{3}}\left(\frac{3}{2} H_{\mathrm{F}}\right)\left(1-\frac{1}{1+e^{\beta\left(d-d_{0}\right)}}\right)\right] l
\end{gathered}
$$

In eq $2, H_{\mathrm{N}}$ and $H_{\mathrm{F}}$ are the Hamaker coefficients in the near and far limit of the sephacryl surface separation from the SWNT, respectively, and $\beta$ and $d_{0}$ are blending terms accounting for the transition between short- and long-range interactions.

We expand this definition by incorporating an electrostatic repulsive force between the anionic amide on the sephacryl surface and the SWNT. ${ }^{46}$ We approximate that, on the order of the SWNT-sephacryl binding site interaction length, the anionic charge on the sephacryl surface will be uniformly distributed, which we denote as $\sigma_{\mathrm{SEPH}}$. Furthermore, the SWNT is also approximated to exhibit a uniform charge density along its cylindrical surface, denoted as $\sigma_{\text {SWNT }}$ with radius $r_{\text {SWNT }}$ and length $l$. The sephacryl-SWNT system is immersed in a liquid with dielectric permittivity $\varepsilon$ and debye length $\kappa$ and represented by Ohshima et al. ${ }^{47}$ as shown in eq 3

$$
\begin{gathered}
V_{\text {electrostatic }}(d)=\varepsilon \varepsilon_{0} \sqrt{\pi \kappa r_{\mathrm{SWNT}}}\left(2 \sqrt{2}\left(\frac{\sigma_{\mathrm{SEPH}}}{\varepsilon \varepsilon_{0} \kappa}\right)\right. \\
\left(\frac{\sigma_{\mathrm{SWNT}}}{\varepsilon \varepsilon_{0} \kappa} \frac{K_{0}\left(\kappa r_{\mathrm{SWNT}}\right)}{K_{1}\left(\kappa r_{\mathrm{SWNT}}\right)}\right) e^{-\kappa d}+\left(\frac{\sigma_{\mathrm{SEPH}}}{\varepsilon \varepsilon_{0} \kappa}\right)^{2} e^{-2 \kappa d} \\
\left.+\left(\frac{\sigma_{\mathrm{SWNT}}}{\varepsilon \varepsilon_{0} \kappa} \frac{K_{0}\left(\kappa r_{\mathrm{SWNT}}\right)}{K_{1}\left(\kappa r_{\mathrm{SWNT}}\right)}\right)^{2} \sqrt{\frac{r_{\mathrm{SWNT}}}{r_{\mathrm{SWNT}}+d}} e^{-2 \kappa d}\right) l
\end{gathered}
$$

where $\varepsilon_{0}$ is the vacuum permittivity and $K_{n}$ is the modified Bessel function of the $n$th kind. We can describe the repulsion between a sephacryl binding site and a SWNT by a hard-sphere model, with a $1 / d^{12}$ distance-dependent repulsion. As such, the total energy of the system is the sum of hard-surface repulsions for SWNT-sephacryl site distances below $2 \AA$, the van der Waals attractions between 2 and $4 \AA$, by electrostatic repulsion between $4 \AA$ and $3 \mathrm{~nm}$, and by thermal energy beyond $3 \mathrm{~nm}$. We can model the kinetics of semiconducting SWNT binding to the sephacryl surface for a given SWNT chirality. We define the rate constant for this process as a distance-dependent energy profile, $V(d)$, for each particle between $d=\infty$ and $d=$ 
Table 1. Chirality-Dependent Effective Linear Charge Density $\xi_{n, m}\left(\mathrm{e}^{-} / \mathrm{nm}\right)$ Based on Our Model That Must Be True in Order to Enable the Separations That We Measure in This Study ${ }^{a}$

\begin{tabular}{llllllllll} 
& $(6,4)$ & $(6,5)$ & $(9,1)$ & $(7,5)$ & $(8,3)$ & $(8,4)$ & $(7,6)$ & $(9,4)$ & $(8,7)$ \\
SDS & 1.29 & 1.77 & 1.77 & 2.24 & 1.72 & 1.91 & 1.64 & 1.71 \\
SDS + SC & 1.29 & 1.87 & 1.77 & 1.93 & 1.72 & 1.78 & 1.70 & 1.71 \\
SDS + STC & 1.29 & 2.10 & 1.50 & 2.62 & 1.77 & 2.41 & 1.82 & 1.26 & 1.47 \\
\hline
\end{tabular}

${ }^{a}$ We only report the chiralities for which there is a measurable change in the rate constant and therefore the charge density around the tube.
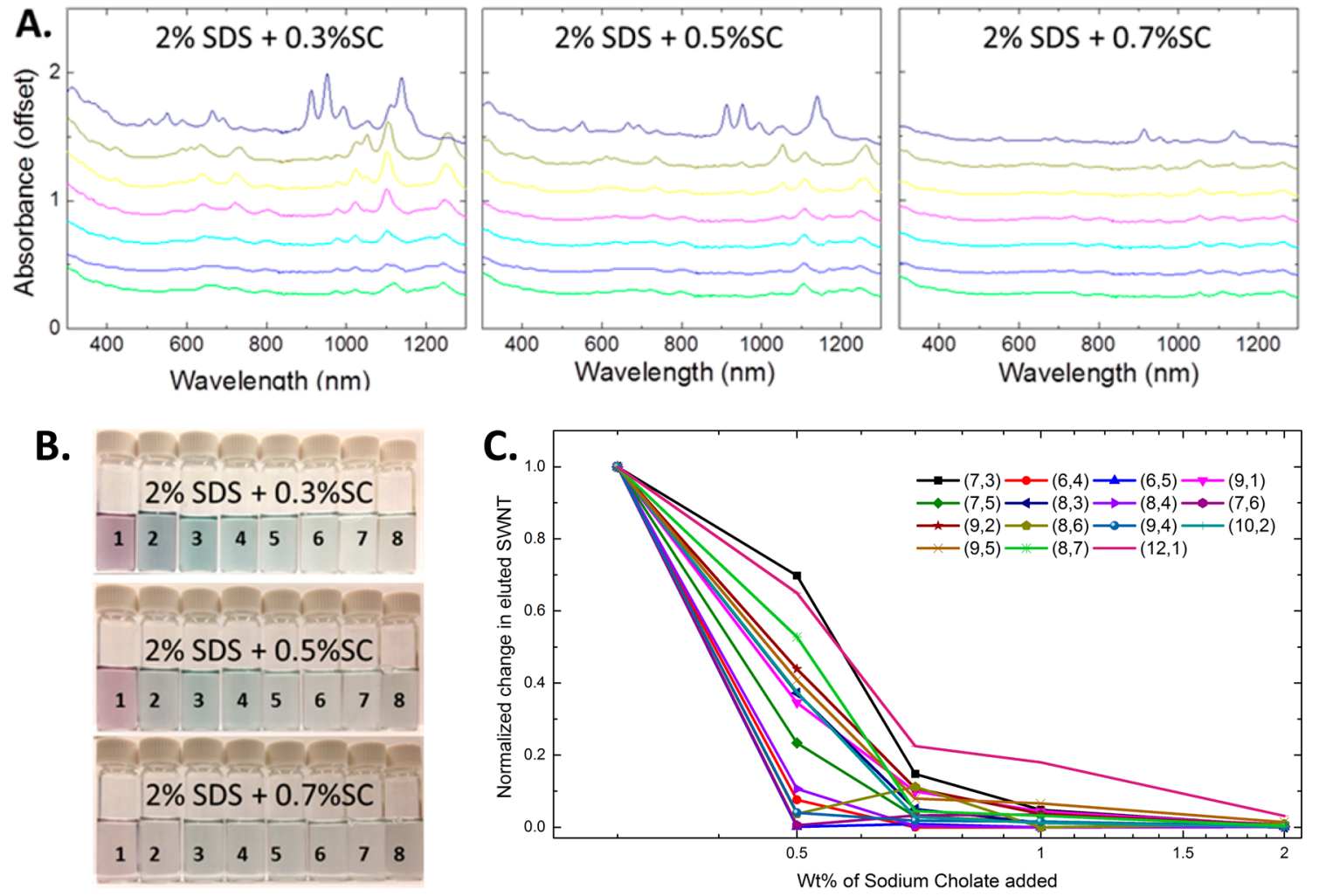

Figure 5. (A) From left to right and top to bottom, the eluted SWNT absorbance spectra of several columns (column 1 on top, column 8 at the bottom) of SWNTs suspended in 2 wt \% SDS with the incremental addition of sodium cholate. (B) From top to bottom, pictures of the eluted samples with incremental addition of sodium cholate, showing an obvious reduction in SWNTs as SC fraction is increased. (C) Change in the quantity of SWNTs eluted in column 2 (a representative column) of the separation as the SC fraction is increased. The change is normalized to the quantity eluted in the $0.3 \%$ SC case (i.e., the lowest fractional addition of SC). Note that the plot is a semilog plot for clarity. Interestingly, the change in number of SWNTs eluted is chirality dependent as the SC concentration is increased. Also, at the point at which $2 \%$ SC is added, there is no detectable SWNT in the solutions.

$\mathrm{d}_{\text {bound }}$ using a relationship for a particle traversing such an energy profile developed by Fuchs et al. ${ }^{48,49}$ In this equation, $k_{\mathrm{b}}$ is the Boltzmann constant, $T$ the system temperature, and $D$ the SWNT diffusion coefficient as approximated by an Einstein-Smoluchowski relation for a cylinder in solution. ${ }^{50,51}$

$$
k_{\mathrm{f}}=\frac{4 \pi \mathrm{D}}{\int_{d_{\mathrm{bound}}}^{\infty} \frac{1}{d^{2}} e^{V(d) / k_{\mathrm{b}} T} d d}
$$

For this particular model, we consider the contributions not only of the system equilibrated in SDS but also in the mixed surfactant system and the effective linear charge density $\xi_{n, m}$ $\left(\mathrm{e}^{-} / \mathrm{nm}\right)$ of SDS + SC and SDS + STC mixed surfactant systems (Table 1). We fit rate constants for each chirality under each surfactant mixture and found effective linear charge densities, $\xi_{n, m}\left(\mathrm{e}^{-} / \mathrm{nm}\right)$, for each chirality under the addition of sodium cholate and sodium taurocholate. Table 1 shows the chiralities that had a measurable change in the charge density based on our model. As we expect, all fall within our previously determined range of $0.41-3.32 \mathrm{e}^{-} / \mathrm{nm}$. Further, the chiralities that elute sooner show a reduced linear charge density. For example, the $(9,1)$ tube shows a significant decrease in effective charge upon STC addition (from 1.77 to $1.50 \mathrm{e}^{-} / \mathrm{nm}$ ), thereby enabling greater adsorption and elution as observed. Note that there is no predicted change between SDS and SDS + SC. Similarly, the $(6,5)$ SWNT decreases in its ability to be separated with an increase in charge density (from 1.77 to 1.87 $\mathrm{e}^{-} / \mathrm{nm}$ ) upon SC addition. As such, our previously established theory appears self-consistent. However, we recognize that we are unable to provide insight into the fractional coverage of one surfactant vs another via this theory.

In order to study the competitiveness of the surfactant upon mixing, we carry out experiments where we sonicate 2 wt \% SDS with the SWNT. We then add an incrementally increasing fraction of sodium cholate in adsorption; however, we still elute the sample with 5\% SDS as discussed in the SI methods section. We perform the separation for eight columns and the absorption spectra are shown in Figure 5(A). 
As we expect, as we increase SC percentage in the bulk solution we reduce the amount of SWNTs that bind to the gel as the SC outcompetes the SDS for tight coverage around the tube, eventually leaving no exposed surface to bind with the gel. As others have noted in density gradient or two-phase separation methods, changing the ratio of SC to SDS changes the amount of the surface of the SWNT that is exposed. Using this understanding is instrumental to being able to tailor either the gel-based separation or other methods, especially the density gradient and two-phase methods which use precisely these two surfactants and in these ratio ranges. The field still does not have a good way to visualize the precise structure of the surfactant on the tube when in solution. However, we can say with certainty that the SC binds tightly with the SWNT and that as the concentration of SC increases to $2 \%$ it outcompetes the SDS coverage on the SWNT.

What is interesting to note is that the binding competition between SC and SDS is once again chirality dependent. We fit the peaks for each of the spectra and observe the changes in the amount of each chirality of nanotubes eluted as the sodium cholate concentration increases. Figure 5(C) shows the change in quantities of each chirality for the second column we elute as the SC concentration is increased. This plot makes it clear that the fractional change is more dramatic for some chiralities than others. The change seems to be the least for larger diameter tubes and the most for smaller diameter tubes. This ordering is similar to the saturation ordering of SDS on a SWNT (larger diameter SWNT saturates the earliest). This observation is consistent with our previously defined model of SDS packing on the tube, ${ }^{1}$ as at $2 \%$ SDS we expect that the smaller diameter tubes are less saturated and hence have the ability to accommodate more SC and cause a dramatic difference in the fractional binding and elution of those tubes. Of specific interest is the $(6,5)$ chirality, which sees a very large change from 0.3 to $0.5 \%$ SC, indicating that the loose structure of SDS at $2 \%$ is easily outcompeted at even lower concentrations of SC. What is surprising, however, is that the $(7,3)$ tube seems to change very slowly with increasing SC concentration, even though we know that the SDS packing is not saturated on the $(7,3)$ tube. This response implies that even the SC surfactant does not have a strong affinity for this tube. It is important to remember that for each chirality we have to consider the competitive binding individually. Our model suggests that the surfactant-bound nanotubes, and their binding to the sephacryl gel, are a kinetically controlled process. Changing the concentration of cosurfactant will affect each nanotube chirality differently. Differences in cosurfactant ratio drive the fractional yield to vary significantly, which is tunable for kinetically driven binding and chirality-specific elutions. Each surfactant will have a binding affinity that is chirality dependent, and hence competitive binding on the tube surface must be considered for each chirality separately.

We conduct a similar experiment for SDOC and STC where we added $0.3 \%$ and $0.7 \%$ by weight of each of these surfactants and measure the absorbance spectra in each case, as is shown in Figure S1. We note once again that SDOC binds very tightly at even low concentrations and do not observe any eluted SWNT. Further, we note that for STC, as was the case for the SC, the biggest change from $0.3 \%$ to $0.7 \%$ is the absolute concentrations for each of the eluted chiralities. Given the low concentrations of SWNT eluted with STC, we were unable to reliably quantify the per chirality difference in binding over the noise levels in the spectra. Nevertheless, our experiments give the first quantitative insight into the relative competitiveness of SDS and SC in a chirality-dependent fashion, and this system is currently the most important for SWNT separation in both the density gradient and two-phase separation methods.

\section{CONCLUSIONS}

All of our observations point to the same conclusion that mixed surfactant systems can be used and controlled to carefully regulate and modify the separation of SWNTs using any of the popular processes in the literature today. Importantly, we show that SC and SDS compete within concentration ranges of $0-$ $2 \%$ each and have a strong chirality dependence. We also show that with STC there are ways to specifically modify the separation and obtain only specific chiralities, which are likely the chiralities to which STC binds least strongly. We expect that our findings will enable further understanding of SWNT surfactant systems and their importance in the emerging field of chirality separation of SWNT.

\section{ASSOCIATED CONTENT}

\section{S Supporting Information}

The Supporting Information is available free of charge on the ACS Publications website at DOI: 10.1021/acs.jpcc.5b07947.

Details of the experimental methods used for this study, absorbance measurements, and analysis of the elution from the separations with bile salt surfactants added (PDF)

\section{AUTHOR INFORMATION}

\section{Corresponding Author}

*E-mail: strano@mit.edu.

\section{Notes}

The authors declare no competing financial interest.

\section{ACKNOWLEDGMENTS}

RMJ gratefully acknowledges support from the National Science Foundation Graduate Research Fellowship and the Department of Defense through the National Defense Science and Engineering Graduate Fellowship. MPL acknowledges an NSF postdoctoral research fellowship under award no. 1306229, a NARSAD Young Investigator Award, and a Burroughs Wellcome Fund Career Award at the Scientific Interface (CASI). M.S.S. acknowledges a grant from the National Science Foundation for the support of this work.

\section{REFERENCES}

(1) Jain, R. M.; Tvrdy, K.; Han, R.; Ulissi, Z.; Strano, M. S. Quantitative Theory of Adsorptive Separation for the Electronic Sorting of Single-Walled Carbon Nanotubes. ACS Nano 2014, 8, 3367-3379.

(2) Tvrdy, K.; Jain, R. M.; Han, R.; Hilmer, A. J.; McNicholas, T. P.; Strano, M. S. A Kinetic Model for the Deterministic Prediction of GelBased Single-Chirality Single-Walled Carbon Nanotube Separation. ACS Nano 2013, 7, 1779-1789.

(3) Arnold, M. S.; Green, A. A.; Hulvat, J. F.; Stupp, S. I.; Hersam, M. C. Sorting Carbon Nanotubes by Electronic Structure Using Density Differentiation. Nat. Nanotechnol. 2006, 1, 60-65.

(4) Green, A. A.; Duch, M. C.; Hersam, M. C. Isolation of SingleWalled Carbon Nanotube Enantiomers by Density Differentiation. Nano Res. 2009, 2, 69-77.

(5) Green, A. A.; Hersam, M. C. Nearly Single-Chirality SingleWalled Carbon Nanotubes Produced Via Orthogonal Iterative Density Gradient Ultracentrifugation. Adv. Mater. 2011, 23, 2185-2190. 
(6) Jain, R. M.; Howden, R.; Tvrdy, K.; Shimizu, S.; Hilmer, A. J.; McNicholas, T. P.; Gleason, K. K.; Strano, M. S. Polymer-Free nearInfrared Photovoltaics with Single Chirality $(6,5)$ Semiconducting Carbon Nanotube Active Layers. Adv. Mater. 2012, 24, 4436-4439.

(7) Zheng, M.; Jagota, A.; Semke, E. D.; Diner, B. A.; McLean, R. S.; Lustig, S. R.; Richardson, R. E.; Tassi, N. G. DNA-Assisted Dispersion and Separation of Carbon Nanotubes. Nat. Mater. 2003, 2, 338-342.

(8) Zheng, M.; Jagota, A.; Strano, M. S.; Santos, A. P.; Barone, P.; Chou, G.; Diner, B. A.; Dresselhaus, M. S.; Mclean, R. S.; Onoa, G. B.; et al. Structure-Based Carbon Nanotube Sorting by SequenceDependent DNA Assembly. Science 2003, 302, 1545-1548.

(9) Khripin, C. Y.; Fagan, J. A.; Zheng, M. Spontaneous Partition of Carbon Nanotubes in Polymer-Modified Aqueous Phases. J. Am. Chem. Soc. 2013, 135, 6822-6825.

(10) Fagan, J. A.; Khripin, C. Y.; Silvera Batista, C. A.; Simpson, J. R.; Hároz, E. H.; Hight Walker, A. R.; Zheng, M. Isolation of Specific Small-Diameter Single-Wall Carbon Nanotube Species Via Aqueous Two-Phase Extraction. Adv. Mater. 2014, 26, 2800-2804.

(11) Liu, H.; Tanaka, T.; Urabe, Y.; Kataura, H. High-Efficiency Single-Chirality Separation of Carbon Nanotubes Using TemperatureControlled Gel Chromatography. Nano Lett. 2013, 13, 1996-2003.

(12) Liu, H. P.; Nishide, D.; Tanaka, T.; Kataura, H. Large-Scale Single-Chirality Separation of Single-Wall Carbon Nanotubes by Simple Gel Chromatography. Nat. Commun. 2011, 2, 1-8.

(13) Blanch, A. J.; Quinton, J. S.; Shapter, J. G. The Role of Sodium Dodecyl Sulfate Concentration in the Separation of Carbon Nanotubes Using Gel Chromatography. Carbon 2013, 60, 471-480.

(14) Gui, H.; Li, H. B.; Tan, F. R.; Jin, H. H.; Zhang, J.; Li, Q. W. Binary Gradient Elution of Semiconducting Single-Walled Carbon Nanotubes by Gel Chromatography for Their Separation According to Chirality. Carbon 2012, 50, 332-335.

(15) Tulevski, G. S.; Franklin, A. D.; Afzali, A. High Purity Isolation and Quantification of Semiconducting Carbon Nanotubes Via Column Chromatography. ACS Nano 2013, 7, 2971-2976.

(16) Sanchez-Valencia, J. R.; Dienel, T.; Groning, O.; Shorubalko, I.; Mueller, A.; Jansen, M.; Amsharov, K.; Ruffieux, P.; Fasel, R. Controlled Synthesis of Single-Chirality Carbon Nanotubes. Nature 2014, 512, 61-64.

(17) Giraldo, J. P.; Landry, M. P.; Faltermeier, S. M.; McNicholas, T. P.; Iverson, N. M.; Boghossian, A. A.; Reuel, N. F.; Hilmer, A. J.; Sen, F.; Brew, J.; et al. Plant Nanobionics Approach to Augment Photosynthesis and Biochemical Sensing. Nat. Mater. 2014, 13, 400-408.

(18) Diao, S.; Hong, G.; Robinson, J. T.; Jiao, L.; Antaris, A. L.; Wu, J. Z.; Choi, C. L.; Dai, H. Chirality Enriched $(12,1)$ and $(11,3)$ SingleWalled Carbon Nanotubes for Biological Imaging. J. Am. Chem. Soc. 2012, 134, 16971-16974.

(19) Jain, A.; Homayoun, A.; Bannister, C. W.; Yum, K. SingleWalled Carbon Nanotubes as near-Infrared Optical Biosensors for Life Sciences and Biomedicine. Biotechnol. J. 2015, 10, 447-459.

(20) Giraldo, J. P.; Landry, M. P.; Kwak, S. Y.; Jain, R. M.; Wong, M. H.; Iverson, N. M.; Ben-Naim, M.; Strano, M. S. A Ratiometric Sensor Using Single Chirality Near-Infrared Fluorescent Carbon Nanotubes: Application to In Vivo Monitoring. Small 2015, 11, 3973-3984.

(21) Kruss, S.; Landry, M. P.; Vander Ende, E.; Lima, B. M.; Reuel, N. F.; Zhang, J.; Nelson, J.; Mu, B.; Hilmer, A.; Strano, M. Neurotransmitter Detection Using Corona Phase Molecular Recognition on Fluorescent Single-Walled Carbon Nanotube Sensors. J. Am. Chem. Soc. 2014, 136, 713-724.

(22) Zhang, J. Q.; Landry, M. P.; Barone, P. W.; Kim, J.; Lin, S.; Ulissi, Z. W.; Lin, D.; Mu, B.; Boghossian, A. A.; Hilmer, A. J.; et al. Molecular Recognition Using Corona Phase Complexes Made of Synthetic Polymers Adsorbed on Carbon Nanotubes. Nat. Nanotechnol. 2013, 8, 959-968.

(23) Nelson, J. T.; Kim, S.; Reuel, N. F.; Salem, D. P.; Bisker, G.; Landry, M. P.; Kruss, S.; Barone, P. W.; Kwak, S.; Strano, M. S. Mechanism of Immobilized Protein A Binding to Immunoglobulin G on Nanosensor Array Surfaces. Anal. Chem. 2015, 87, 8186-8193.
(24) Landry, M. P.; Vukovic, L.; Kruss, S.; Bisker, G.; Landry, A. M.; Islam, S.; Jain, R.; Schulten, K.; Strano, M. S. Comparative Dynamics and Sequence Dependence of DNA and RNA Binding to Single Walled Carbon Nanotubes. J. Phys. Chem. C 2015, 119, 10048-10058.

(25) Wang, Q. H.; Bellisario, D. O.; Drahushuk, L. W.; Jain, R. M.; Kruss, S.; Landry, M. P.; Mahajan, S. G.; Shimizu, S. F. E.; Ulissi, Z. W.; Strano, M. S. Low Dimensional Carbon Materials for Applications in Mass and Energy Transport. Chem. Mater. 2014, 26, 172-183.

(26) Bindl, D. J.; Arnold, M. S. Efficient Exciton Relaxation and Charge Generation in Nearly Monochiral $(7,5)$ Carbon Nanotube/ C60 Thin-Film Photovoltaics. J. Phys. Chem. C 2013, 117, 2390-2395.

(27) LeMieux, M. C.; Roberts, M.; Barman, S.; Jin, Y. W.; Kim, J. M.; Bao, Z. N. Self-Sorted, Aligned Nanotube Networks for Thin-Film Transistors. Science 2008, 321, 101-104.

(28) Avouris, P.; Freitag, M.; Perebeinos, V. Carbon-Nanotube Photonics and Optoelectronics. Nat. Photonics 2008, 2, 341-350.

(29) Yu, D.; Liu, H.; Peng, L.-M.; Wang, S. Flexible Light-Emitting Devices Based on Chirality-Sorted Semiconducting Carbon Nanotube Films. ACS Appl. Mater. Interfaces 2015, 7, 3462-3467.

(30) Wang, H.; Koleilat, G. I.; Liu, P.; Jiménez-Osés, G.; Lai, Y.-C.; Vosgueritchian, M.; Fang, Y.; Park, S.; Houk, K. N.; Bao, Z. High-Yield Sorting of Small-Diameter Carbon Nanotubes for Solar Cells and Transistors. ACS Nano 2014, 8, 2609-2617.

(31) Clar, J. G.; Silvera Batista, C. A.; Youn, S.; Bonzongo, J.-C. J.; Ziegler, K. J. Interactive Forces between Sodium Dodecyl SulfateSuspended Single-Walled Carbon Nanotubes and Agarose Gels. J. Am. Chem. Soc. 2013, 135, 17758-17767.

(32) Rauwald, U.; Shaver, J.; Klosterman, D. A.; Chen, Z. Y.; SilveraBatista, C.; Schmidt, H. K.; Hauge, R. H.; Smalley, R. E.; Ziegler, K. J. Electron-Induced Cutting of Single-Walled Carbon Nanotubes. Carbon 2009, 47, 178-185.

(33) Chen, Y. S.; Xu, Y. Q.; Wang, Q. M.; Gunasinghe, R. N.; Wang, X. Q.; Pang, Y. Highly Selective Dispersion of Carbon Nanotubes by Using Poly(Phenyleneethynylene)-Guided Supermolecular Assembly. Small 2013, 9, 870-875.

(34) Chen, Y. S.; Xu, Y. Q.; Perry, K.; Sokolov, A. P.; More, K.; Pang, Y. Achieving Diameter-Selective Separation of Single-Walled Carbon Nanotubes by Using Polymer Conformation-Confined Helical Cavity. ACS Macro Lett. 2012, 1, 701-705.

(35) Hirano, A.; Tanaka, T.; Urabe, Y.; Kataura, H. pH- and SoluteDependent Adsorption of Single-Wall Carbon Nanotubes onto Hydrogels: Mechanistic Insights into the Metal/Semiconductor Separation. ACS Nano 2013, 7, 10285-10295.

(36) Flavel, B. S.; Kappes, M. M.; Krupke, R.; Hennrich, F. Separation of Single-Walled Carbon Nanotubes by 1-DodecanolMediated Size-Exclusion Chromatography. ACS Nano 2013, 7, 35573564.

(37) Silvera-Batista, C. A.; Scott, D. C.; McLeod, S. M.; Ziegler, K. J. A Mechanistic Study of the Selective Retention of Sds-Suspended Single-Wall Carbon Nanotubes on Agarose Gels. J. Phys. Chem. C 2011, 115, 9361-9369.

(38) Flavel, B. S.; Moore, K. E.; Pfohl, M.; Kappes, M. M.; Hennrich, F. Separation of Single-Walled Carbon Nanotubes with a Gel Permeation Chromatography System. ACS Nano 2014, 8, 1817-1826.

(39) Duque, J. G.; Densmore, C. G.; Doorn, S. K. Saturation of Surfactant Structure at the Single-Walled Carbon Nanotube Surface. J. Am. Chem. Soc. 2010, 132, 16165-16175.

(40) Tanaka, T.; Urabe, Y.; Nishide, D.; Kataura, H. Discovery of Surfactants for Metal/Semiconductor Separation of Single-Wall Carbon Nanotubes Via High-Throughput Screening. J. Am. Chem. Soc. 2011, 133, 17610-17613.

(41) Nair, N.; Kim, W. J.; Braatz, R. D.; Strano, M. S. Dynamics of Surfactant-Suspended Single-Walled Carbon Nanotubes in a Centrifugal Field. Langmuir 2008, 24, 1790-1795.

(42) Ghosh, S.; Bachilo, S. M.; Weisman, R. B. Advanced Sorting of Single-Walled Carbon Nanotubes by Nonlinear Density-Gradient Ultracentrifugation. Nat. Nanotechnol. 2010, 5, 443-450.

(43) Subbaiyan, N. K.; Cambré, S.; Parra-Vasquez, A. N. G.; Hároz, E. H.; Doorn, S. K.; Duque, J. G. Role of Surfactants and Salt in 
Aqueous Two-Phase Separation of Carbon Nanotubes toward Simple Chirality Isolation. ACS Nano 2014, 8, 1619-1628.

(44) Hilmer, A. J.; McNicholas, T. P.; Lin, S.; Zhang, J.; Wang, Q.; Mendenhall, J. D.; Song, C.; Heller, D. A.; Barone, P. W.; Blankschtein, D.; et al. Role of Adsorbed Surfactant in the Reaction of Aryl Diazonium Salts with Single-Walled Carbon Nanotubes. Langmuir 2012, 28, 1309-1321.

(45) Rajter, R. F.; Podgornik, R.; Parsegian, V. A.; French, R. H.; Ching, W. Y. Van Der Waals-London Dispersion Interactions for Optically Anisotropic Cylinders: Metallic and Semiconducting SingleWall Carbon Nanotubes. Phys. Rev. B: Condens. Matter Mater. Phys. 2007, 76, 1-16.

(46) White, B.; Banerjee, S.; O’Brien, S.; Turro, N. J.; Herman, I. P. Zeta-Potential Measurements of Surfactant-Wrapped Individual Single-Walled Carbon Nanotubes. J. Phys. Chem. C 2007, 111, 13684-13690.

(47) Ohshima, H. Electrostatic Interaction between a Cylinder and a Planar Surface. Colloid Polym. Sci. 1999, 277, 563-569.

(48) Jeng, E. S.; Shih, C. J.; Barone, P. W.; Jones, N.; Baik, J. H.; Abrahamson, J. T.; Strano, M. S. A Compositional Window of Kinetic Stability for Amphiphilic Polymers and Colloidal Nanorods. J. Phys. Chem. C 2011, 115, 7164-7170.

(49) Fuchs, N. Z. Phys. Chem.-Abteilung a-Chem. Thermodyn. Kinetik Elektrochem. Eigenschaftslehre 1934, 4, 171-175.

(50) Bloomfield, V.; Dalton, W. O.; Vanholde, K. E. Frictional Coefficients of Multisubunit Structures.I. Theory. Biopolymers 1967, 5, 135-148.

(51) Incropera, F. P.; DeWitt, D. P. Fundamentals of Heat and Mass Transfer, 5th ed; John Wiley \& Sons: Hoboken, NJ, 2002. 